\title{
Temporal Response of Endogenous Neural Progenitor Cells Following Injury to the Adult Rat Spinal Cord
}

\author{
Yilin Mao, Kathryn Mathews and Catherine A. Gorrie* \\ Neural Injury Research Unit, School of Life Sciences, Faculty of Science, University of Technology Sydney, \\ Sydney, NSW, Australia
}

A pool of endogenous neural progenitor cells (NPCs) found in the ependymal layer and the sub-ependymal area of the spinal cord are reported to upregulate Nestin in response to traumatic spinal cord injury (SCl). These cells could potentially be manipulated within a critical time period offering an innovative approach to the repair of SCl. However, little is known about the temporal response of endogenous NPCs following SCl. This study used a mild contusion injury in rat spinal cord and immunohistochemistry to determine the temporal response of ependymal NPCs following injury and their correlation to astrocyte activation at the lesion edge. The results from the study demonstrated that Nestin staining intensity at the central canal peaked at $24 \mathrm{~h}$ post-injury and then gradually declined over time. Reactive astrocytes double labeled by Nestin and glial fibrillary acidic protein (GFAP) were found at the lesion edge and commenced to form the glial scar from 1 week after injury. We conclude that the critical time period for manipulating endogenous NPCs following a spinal cod injury in rats is between $24 \mathrm{~h}$ when Nestin expression in ependymal cells is increased and 1 week when astrocytes are activated in large numbers.

\footnotetext{
Keywords: spinal cord injury, neural progenitor cells, reactive astrocytes, astrogliosis, temporal response, critical time period, Nestin, GFAP
}

\section{INTRODUCTION}

Traumatic spinal cord injury (SCI) arises from a mechanical insult that damages the spinal cord. People living with SCI have symptoms that can include a range of sensory and motor deficits, chronic pain, bladder/bowel dysfunction as well as autonomic and sexual dysfunction (Norenberg et al., 2004). These reductions in function can affect quality of life for those suffering from the injury, and can also have an impact on society, with the families and the community, bearing many of the emotional and financial costs of SCI. At present, there are no fully restorative treatments for SCI, although various molecular, cellular and rehabilitative therapies have been approaching clinical trials and have shown a limited degree of functional recovery. Unfortunately, the existing clinical approaches for SCI are still limited in the short term (Thuret et al., 2006), with long-term functional outcomes largely unchanged despite the distinct improvement in the acute mortality rate (DeVivo, 2007). Consequently, it is critical to develop novel approaches for long-term functional recovery from SCI. Manipulation of neural progenitor cells (NPCs) is a promising avenue of approach.

NPCs are multipotent and able to differentiate into neurons, oligodendrocytes and astrocytes in vitro (Dromard et al., 2008). They are capable of self-renewal, proliferation and 
differentiation, although these responses are more limited and weaker compared with pluripotent stem cells (Mothe et al., 2011). NPCs are found throughout the mammalian central nervous system (CNS), including the spinal cord (Mothe and Tator, 2005; Mothe et al., 2011). These cells show promising results in a range of animal models investigating functional recovery after SCI (Mothe and Tator, 2005; Cizkova et al., 2009; Mothe et al., 2011; Faulkner et al., 2013). There are two principal ways in which NPCs may be used; exogenous NPCs can be transplanted into an injury site, or endogenous NPCs could be manipulated in situ for spinal cord repair. Both approaches, however, focus on utilizing the neuroregenerative capacity of NPCs to replace damaged or lost cells in the injured spinal cord. The manipulation of endogenous NPCs as a minimally invasive therapy may be preferable for treating SCI, because it can circumvent key issues associated with stem cell transplant, such as immune responses and cell rejection, tumorigenesis and ethical issues regarding the sourcing and transplanting of stem cells (Barreiro-Iglesias, 2010).

In the normal uninjured spinal cord, endogenous NPCs are quiescent in the ependymal and sub-ependymal areas of the central canal (Shibuya et al., 2002; Hamilton et al., 2009). However following injury endogenous NPCs in the spinal cord proliferate, migrating to the lesion site for differentiation (Hofstetter et al., 2005; Horky et al., 2006; Meletis et al., 2008; Cizkova et al., 2009; Barnabé-Heider et al., 2010). After migration, the majority of the activated NPCs differentiate into astrocytes, contributing to glial scar formation (Ke et al., 2006; Vessal et al., 2007; Barnabé-Heider et al., 2010). This glial scar, while protecting the injury site from further damage, also inhibits the repair of the injury by creating a physical and chemical barrier. Spinal cord NPCs are capable of differentiation into all three neural lineages, neurons, oligodendrocytes and astrocytes in vitro (Blasko et al., 2012), but there is currently no evidence of spontaneous neurogenesis from endogenous NPCs in vivo (Ke et al., 2006; Vessal et al., 2007). NPCs can differentiate into neurons in vivo given the right conditions as shown by a recent murine study that introduced the transcription factor Sox11 gene via a lentiviral vector (Guo et al., 2014). This treatment pushed NPCs towards a neuronal lineage, increased brain-derived neurotrophic factor expression and resulted in improved functional outcomes. Sox11 activation is associated with an up-regulation of Nestin protein in ependymal cells following SCI. Nestin, a type VI intermediate filament protein, predominately presents in CNS progenitor cells (Tzeng, 2002), and is also expressed by the ependymal cells in the central canal of developing spinal cord and in response to traumatic injury (Sakakibara et al., 2007; Cizkova et al., 2009). In our study, NPCs were recognized by the immunohistochemistry antibody, anti-Nestin, with their histological location around the central canal (Cizkova et al., 2009). We have shown that Nestin is increased in human spinal cords following injury to the CNS, (Cawsey et al., 2015), but it can be difficult to determine the temporal sequences using human autopsy tissue, with all its inherent variation. Although several studies have reported increased Nestin expression in rat models of SCI (Namiki and Tator, 1999; Shibuya et al., 2002; Mothe and Tator, 2005; Horky et al., 2006; Cizkova et al., 2009; Foret et al., 2010), there remains some uncertainty about the time of peak response. We propose that a therapeutic window exists while NPCs are expressing Nestin but before cells are committed to astrocyte differentiation.

\section{MATERIALS AND METHODS}

\section{Animals}

Twenty one adult female Sprague-Dawley rats $(250 \pm 10 \mathrm{~g})$ were used (Animal Resources Centre, Perth, Australia). Rats were maintained in standard cages with ad-lib water and food on a 12:12 h light-dark cycle. Rats underwent SCI, and were euthanased at different time points post-injury, $24 \mathrm{~h}, 1$ week, 2 weeks and 6 weeks. Three rats were subjected to sham surgery (laminectomy only) and kept for 6 weeks following surgery. Six normal rats were sacrificed at $0 \mathrm{~h}$ and 6 weeks as controls ( $n=3$ for all groups). All animal procedures were in accordance with the guidelines of the National Health and Medical Research Council of Australia, and the experimental protocol was approved by The University of Technology Sydney Animal Care and Ethics Committee.

\section{Surgical Procedures}

Anesthesia was induced using 4\% Isofluorane with $1 \mathrm{~L} / \mathrm{min}$ oxygen and then maintained at $2 \%$ Isofluorane with $1 \mathrm{~L} / \mathrm{min}$ oxygen. Skin and muscle were incised and retracted through the dorsal midline and a T10 laminectomy was performed to expose spinal cord. A New York University Weight-Drop Impactor was used to induce a mild contusion SCI $(6.25 \mathrm{~mm}$ drop, $10 \mathrm{~g}$ weight, $2.5 \mathrm{~mm}$ diameter). The surgical data, including compression $(\mathrm{mm})$, compression rate $(\mathrm{m} / \mathrm{s})$ and weight-drop velocity $(\mathrm{m} / \mathrm{s})$, was recorded by the impactor software. The surgical wound was then closed in layers. Rats were given antibiotics (Cephalozin sodium, $33 \mathrm{mg} / \mathrm{kg}$, s.c.), analgesics (Temgesic $0.03 \mathrm{mg} / \mathrm{kg}$, s.c.) and supplementary fluid (Hartman's replacement solution, $15 \mathrm{ml} / \mathrm{kg}$, s.c.) post-operatively twice daily for 3 days. Bladders were expressed manually until the normal voiding response returned. Antibiotics were continued until the urine was clear. The skin suture was removed 7-10 days post-operation.

\section{Behavioral Assessments}

Each animal was assessed for functional recovery on postoperative day 1 only using the BBB Locomotor Rating Scale to ensure consistency of the lesion (Basso et al., 1996). Assessment was carried out by two independent reviewers who were blinded to the groups.

\section{Histology}

Animals were deeply anesthetized by an injection of Lethobarb (Pentobarbitone sodium, $1 \mathrm{ml} / \mathrm{kg}$, i.p.), and then perfused intracardially with $0.9 \%$ saline with heparin, followed by $4 \%$ paraformaldehyde in $0.1 \mathrm{M}$ phosphate ( $\mathrm{pH}$ 7.4). The spinal cord was collected from cervical enlargement to lumbar enlargement for $24 \mathrm{~h}$ fixation in $4 \%$ paraformaldehyde, and then cryoprotected in $30 \%$ sucrose in $0.1 \mathrm{M}$ phosphate buffer with $0.01 \%$ sodium azide. Transverse sections were produced 
at $15 \mu \mathrm{m}$ from a $1 \mathrm{~cm}$ length of spinal cord centered on the injury epicenter. All the sections were mounted in series so that adjacent sections were on consecutive slides. One slide of each spinal cord was stained with Mayer's Haematoxylin and Eosin ( $\mathrm{H} \& \mathrm{E})$ to assess tissue morphology and determine the injury site.

\section{Immunohistochemistry}

One slide of each spinal cord was used for co-labeling of Nestin and glial fibrillary acidic protein (GFAP). Slides were taken through xylene and decreasingly graded ethanol to distilled water. They were then immersed in phosphate buffered saline with $0.2 \%$ Triton X-100 (PBST) at $\mathrm{pH} 7.4$ for $10 \mathrm{~min}$, followed by $5 \%$ normal goat serum in PBST for $30 \mathrm{~min}$. Primary mouse antiNestin (Abcam, 1:1000) and rabbit anti-GFAP (Dako, 1:1000) antibodies were diluted with phosphate buffer with 5\% normal goat serum (PBG), and applied on slides. Primary antibody was omitted on negative control slides. All slides were incubated overnight at $4^{\circ} \mathrm{C}$ in a moist environment. Three changes of PBST were used to wash the slides. The secondary Alexa Fluor fluorescent 568 anti-mouse (Invitrogen, 1:200) and 488 antirabbit (Invitrogen, 1:200) antibodies diluted with PBG were applied on slides. All slides were incubated for $2 \mathrm{~h}$ at room temperature in a moist environment. For nuclear staining, slides were washed by two changes of PBST and then incubated in $0.2 \%$ Hoechst (Invitrogen) for $10 \mathrm{~min}$. All slides were coversliped in fluorescent mounting medium (Dako).

\section{Image Analysis}

All sections were captured using Olympus DP70 camera with the Olympus DP Controller software (Version 3.1.1.267). Entire spinal cord sections were captured at low power $(40 \times)$ and regions of interest (ROI) were also captured at high power $(400 \times)$.

ROI $\left(0.01 \mathrm{~mm}^{2}\right)$ included:

- edge of the lesion;

- center of the lesion;

- ventral white matter and ventral gray matter;

- central canal.

Images were captured on sections containing the lesion epicenter and $2.25 \mathrm{~mm}$ rostral and caudal to the epicenter for each injured spinal cord. The equivalent sections were selected in sham and control groups for imaging.

Injury area was identified as hemeorrhage, axonal swellings, cell death and cavity formation on $\mathrm{H} \& \mathrm{E}$ images. The transverse area of spinal cord and the injured area were measured on every H\&E section using ImageJ Software (National Institutes of Health, Version 2.1.4.7). The injury percentage was calculated as the injured area divided by the transverse area of spinal cord to determine the section containing epicenter of injury (maximum injury percentage).

Nestin and GFAP immunoreactivity was measured in each ROI using the mean gray scale value (GSV) as a measurement of staining intensity. For fluorescent microscopic images the black balance of images was adjusted to the level of negative control as reference by the build-in function of the software (400× magnification, U-MWIG3 filter, $1 / 10 \mathrm{~s}$ exposure time) to control for any variation in staining intensity between sections or staining runs.

\section{Statistics}

Data analysis was performed using GraphPad PRISM Statistics Software (Version 6.0e). All data was expressed as mean \pm standard deviation ( $n=3$ for all groups). Statistically significant differences were considered at $p<0.05$. Pearson's correlations were employed to evaluate the relationship between Nestin and GFAP immunoreactivity. The Pearson's correlation coefficient $(r)$ indicates a linear relationship (Welkowitz et al., 2012). Analysis of variance (ANOVA) was used to compare between the groups and Bonferroni post hoc tests were used to determine significant differences. Paired $t$-tests were used to compare caudal and rostral measurements within in the same animal.

\section{RESULTS}

\section{Contusion Injury}

The mild contusion SCI was produced consistently in all injury animals according to surgical parameters and day 1 BBB scores. There were no significant differences in weight-drop velocity, compression and compression rate between the four injury groups (ANOVA, $p>0.05$ ). The mean weight-drop velocity, compression, and compression rate were $0.348 \pm 0.028 \mathrm{~m} / \mathrm{s}$, $1.707 \pm 0.152 \mathrm{~mm}$ and $0.301 \pm 0.007 \mathrm{~m} / \mathrm{s}$ respectively. In terms of the hindlimb locomotor function at day 1 post-surgery, there were no significant differences in day 1 BBB score between the four injury groups, or between 6-week sham and normal control groups (ANOVA, $p>0.05$ ). The mean BBB scores at day 1 postsurgery were $4.8 \pm 2.5$ for the injury groups and $20.9 \pm 0.2$ for the 6-week sham and normal control groups.

\section{Lesion Morphology}

The mild contusion led to a lesion located in the dorsal surface of the spinal cord (Figure 1A). In injury groups, the central canal at the epicenter of the mild contusion was completely fragmented, and the individual ependymal cells could not be recognized. There was extensive axonal injury in the white matter with axonal swellings and retraction bulbs visible in all injury groups from $24 \mathrm{~h}$ post-injury. Axonal swellings and retraction bulbs are easily identifiable on $\mathrm{H} \& \mathrm{E}$ stained transverse sections of spinal cord (Figure 1B) and appear as small round eosinophilic structures located in the white matter. The lesion site involved necrotic tissue, cellular debris in $24 \mathrm{~h}, 1$-week and 2-week post-injury groups. Hemorrhage was extensive at $24 \mathrm{~h}$ post-injury and reduced by 1 -week following SCI. There were a large number of neutrophils, clearly identifiable by their multilobed nuclei, spreading across the lesion and surrounding area at $24 \mathrm{~h}$ post-injury, suggesting acute inflammation, and they remained in small numbers at 1 week post-injury. Furthermore, the penumbra tissue was included at the edge of lesion in 1 -week and 2 -week post-injury groups. In the 2 -week injury 
A

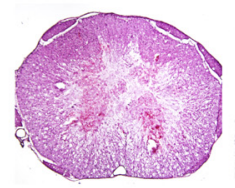

24-Hour

Post-injury

B

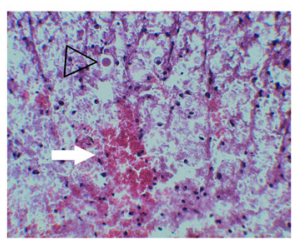

24-Hour

Post-injury

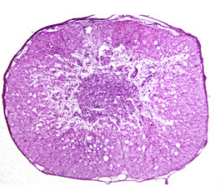

1-Week

Post-injury

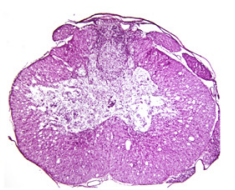

2-Week

Post-injury

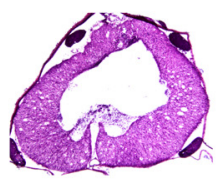

6-Week Post-injury

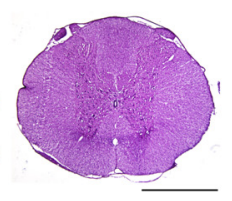

6-Week Sham

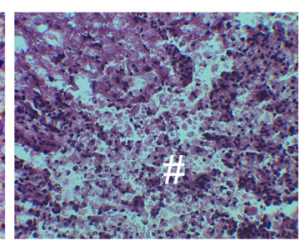

1-Week

Post-injury

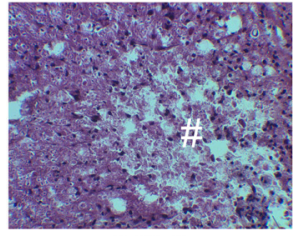

2-Week Post-injury

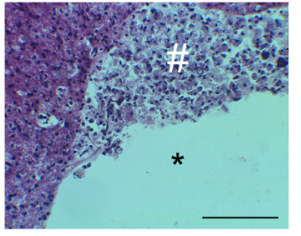

6-Week Post-injury

C

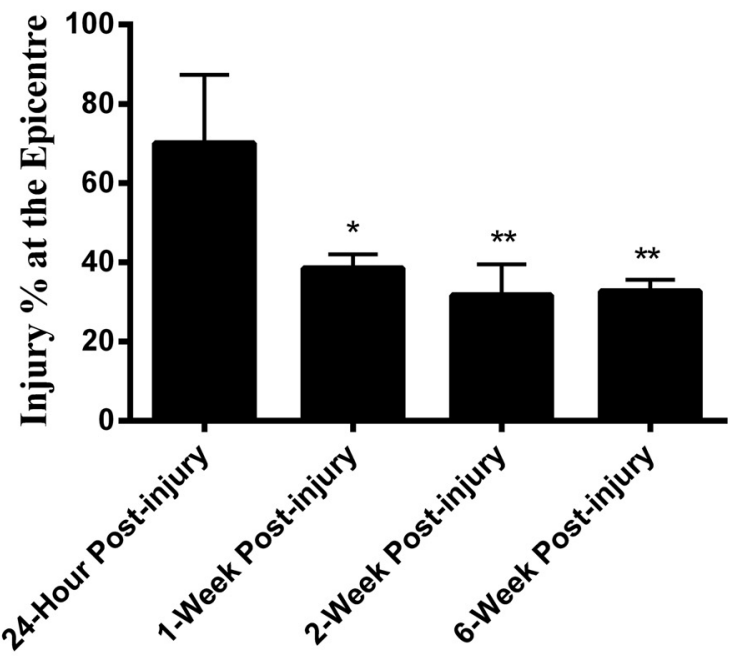

Groups

FIGURE 1 | Lesion epicenter in rat spinal cord transverse sections. (A) Mayer's H\&E staining of lesion epicenter at different time points. The lesion induced by the mild contusion was observed at the dorsal site of the spinal cord in the injury groups. The central canal in the injury groups was destroyed at the epicenter of injury. Scale bar: $500 \mu \mathrm{m}$. (B) Mayer's H\&E staining of lesion area at high power at different time points. Hemorrhage (arrow) was extensive at $24 \mathrm{~h}$ post-injury and reduced by 1 week following spinal cord injury (SCl). Extensive axonal injury with axonal swellings and retraction bulbs (arrow heads) were visible in all injury groups. Necrotic tissue and cellular debris (\#) were observed in the injury groups. Cell loss in the lesion increased over time until a cavity $\left({ }^{*}\right)$ formed by 6-week post-injury. Scale bar: $125 \mu \mathrm{m}$. (C) Percentage of the injured area at the epicenter of injury between injury groups. The $24 \mathrm{~h}$ injury group had a higher injury percentage at the epicenter of injury than other injury groups. Data are presented as mean \pm SD. Statistically significant differences were shown compared to the $24 \mathrm{~h}$ injury group. ${ }^{*} p<0.05,{ }^{* *} p<0.01$.

group, the lesion was a cystic cavity containing cell debris and macrophages. Cell loss in the lesion increased over time until a cavity formed by 6-week post-injury. Furthermore, the irregular shape of spinal cord transverse section was noticed in 6-week post-injury group. In control groups, there were no histological evidences to suggest any lesion in the H\&E sections of sham or normal control spinal cords. Moreover, the H\&E image from the 6-week sham animal was used to represent control spinal cords in Figure 1A.

The injured area as a percentage of total cross sectional area was measured at the epicenter of injury in each spinal cord (Figure 1C). The mean injury percentage at the epicenter of injury ranged from $31.74 \pm 7.83$ to $70.16 \pm 17.24 \%$. There were significant differences in the injury percentage at the epicenter of injury between groups (ANOVA, $p<0.01$ ). The injury percentage at the epicenter of injury in $24 \mathrm{~h}$ post-injury group was significantly higher than those in 1week, 2-week and 6-week post-injury groups (Bonferroni post hoc comparisons, $p<0.05)$. There were no significant differences detected in the percentage of the injured area at the epicenter between 1-week, 2-week and 6-week injury groups. The mean percentage of the injured area at the 

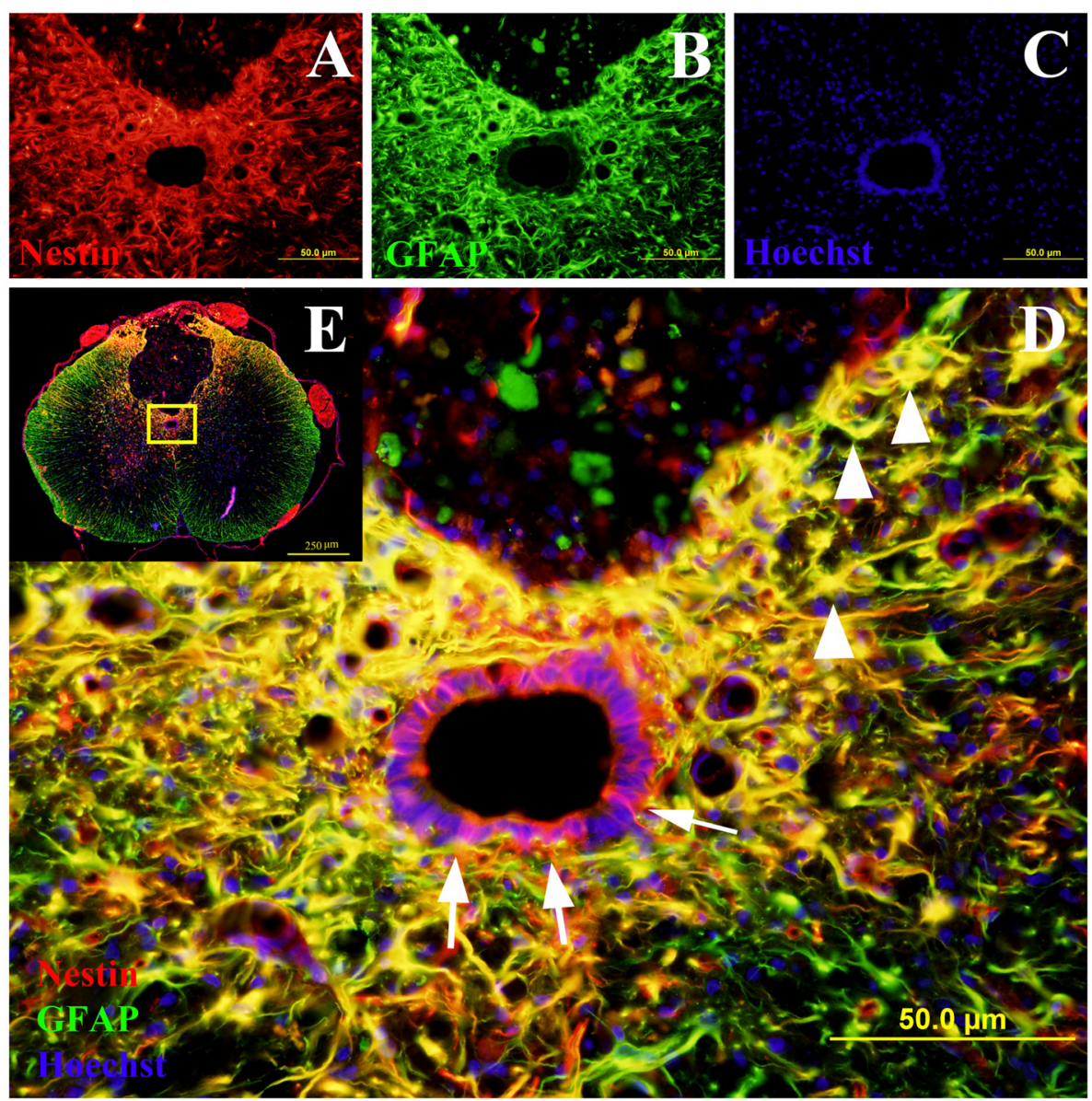

FIGURE 2 | Co-labeling of Nestin and glial fibrillary acidic protein (GFAP) in rat spinal cord transverse sections. The Nestin positive cells were shown in red fluorescence (A), while the GFAP was stained in green fluorescence (B). All cell nuclei were counterstained in blue (C). When the images were merged, the cells co-expressing Nestin and GFAP were shown in yellow at $400 \times$ magnification (D). These study sites (the central canal and the lesion edge) were located in the yellow box area of the spinal cord transverse section at $40 \times$ magnification (E). All the Nestin positive cells at the central canal were not co-labeled with GFAP (arrows). The cells at the lesion edge co-expressed Nestin and GFAP (arrowheads).

epicenter for 1-week, 2-week and 6-week post-injury groups was $34.32 \pm 3.67 \%$.

\section{Cellular Responses}

\section{Nestin}

Nestin positive cells at the central canal consisted of intense cytoplasmic staining of ependymal cells with long basal processes (Figure 2). Nestin positive ependymal cells were seen in all the injury groups and only occasionally in the control groups. There was also increased Nestin staining at the lesion edge in the spinal cords of all the injured groups but not in comparative regions in non-injured controls. Occasional Nestin positive cells were seen in spinal cord blood vessels of all animals regardless of injury status (for example Figure 4A, 6-week sham).

\section{Central Canal}

The quantities of Nestin positive cells at the central canal were compared at different time points following mild contusion SCI in rat, along with sham and control groups (Figure 2). The central canal was identified by the histological morphology of ependymal cells in the round to oval arrangement. The central canal at the epicenter of injury was completely fragmented and not able to be identified due to the mild contusion injury. In injury groups, there were no significant differences in Nestin immunoreactivity at the central canal between rostral and caudal sections. In sham and control groups, the Nestin positivity at the central canal had no significant differences amongst the rostral, epicenter and caudal sections. Therefore, the mean GSV of Nestin immunoreactivity on rostral and caudal sections were used together in injury groups for further analysis, and those on rostral, epicenter and caudal sections were jointly considered in sham and control groups for statistic tests.

The Nestin positivity at the central canal peaked at $24 \mathrm{~h}$ post-injury and decreased with the increasing post-injury time in injury groups (Figure 3). There were significant differences detected in the Nestin immunoreactivity at the central canal between injury groups (ANOVA, $p<0.001$ ) but not sham and 
A

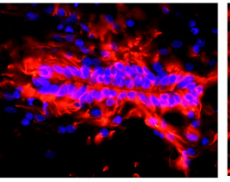

24-Hour

Post-injury

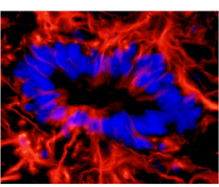

1-Week

Post-injury

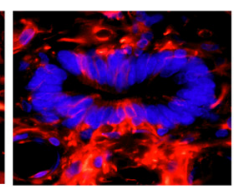

2-Week

Post-injury

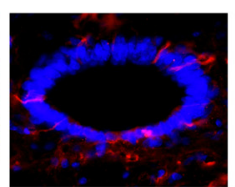

6-Week

Post-injury

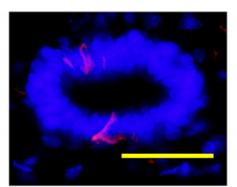

6-Week

Sham

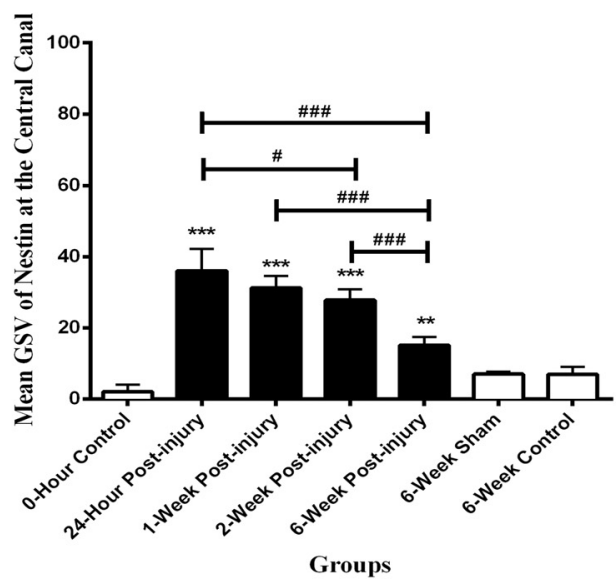

FIGURE 3 | Nestin positive cells at the central canal of rat spinal cord transverse sections. (A) Nestin positive cells were shown in red fluorescence, and the cell nuclei were counterstained in blue. Scale bar: $25 \mu \mathrm{m}$. (B) Nestin immunoreactivity at the central canal of rat spinal cord transverse sections. The Nestin positivity peaked at $24 \mathrm{~h}$ post-injury, followed by a decreased trend in the Nestin immunoreactivity with the increasing post-injury time. Data are presented as mean \pm SD. Statistically significant differences were shown compared to the 6 -week sham group $\left(* * p<0.01,{ }^{* * *} p<0.001\right)$. Statistically significant differences were revealed between groups ( $p<0.05$; ${ }^{\# \# \# ~} p<0.001$ ).

control groups (Bonferroni post hoc comparisons, $p>0.05$ ), so the 6-week control group was used as a standard for all subsequent statistical analysis. The Nestin immunoreactivity in all injury groups were significantly higher than that in 6-week control group (Bonferroni post hoc comparisons, $p<0.05$ ). Moreover, the Nestin immunoreactivity at $24 \mathrm{~h}$ was two-fold higher than that at 6 weeks post-SCI. Counts of ependymal cell nuclei in the central canal epithelium in normal, sham and at different time points post-injury showed no significant differences. This indicates that there was no overall increase in ependymal cell numbers.

\section{Lesion Edge}

The Nestin positivity at the lesion edge was compared between different time points following mild contusion SCI in rat, along with sham and control groups. In sham and control groups, blood vessels were stained as Nestin positive, and a population of cells at the lesion edge in injury groups showed distinct Nestin positivity (Figure 4A).

There were no significant differences between the GSV for Nestin intensity in the rostral, epicenter and caudal sections, thereby an overall GSV was used for statistical analysis. The Nestin positivity at the lesion edge was remarkably upregulated from at 1 week post-injury and continuously increased to the maximum at 6-week post-injury (Figure 4B). The Nestin immunoreactivity at the lesion edge was significantly different between groups (ANOVA, $p<0.001$ ). There were no significant differences in Nestin positivity between sham and control groups (Bonferroni post hoc comparisons, $p>0.05)$, so the 6-week control group was used as a standard for all subsequent statistical analysis. The Nestin positivity in 1-week, 2-week and 6-week post-injury groups was significantly higher than that in 6-week control group respectively (Bonferroni post hoc comparisons, $p<0.001$ ). Furthermore, the 2-week and the 6-week post-injury groups had a significantly larger Nestin positivity compared to 1 -week post-injury groups respectively (Bonferroni post hoc comparisons $p<0.01$ ). The Nestin immunoreactivity at the lesion edge in 6-week post-injury group appeared to be greater than that in 2 -week post-injury group, but not statistically significant (Bonferroni post hoc comparisons, $p>0.05)$.

\section{GFAP}

GFAP positive cells were observed in all the spinal cord sections and typically had the morphology of astrocytes with multiple long processes. White matter regions had increased GFAP staining compared to gray matter in all the control cords. No GFAP staining was seen in ependymal cells lining the central canal in any of the spinal cords.

\section{Lesion Edge}

There was intense GFAP staining of cells at the lesion edge for all injured spinal cords (Figure 4C). GFAP positive cells 
A

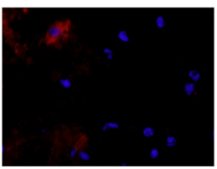

24-Hour

Post-injury

B

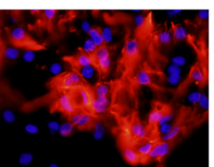

1-Week

Post-injury

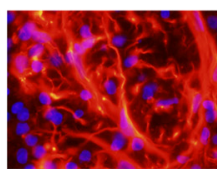

2-Week

Post-injury

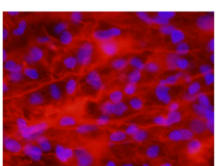

6-Week

Post-injury

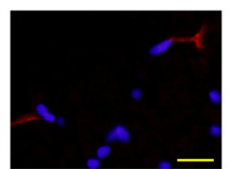

6-Week

Sham

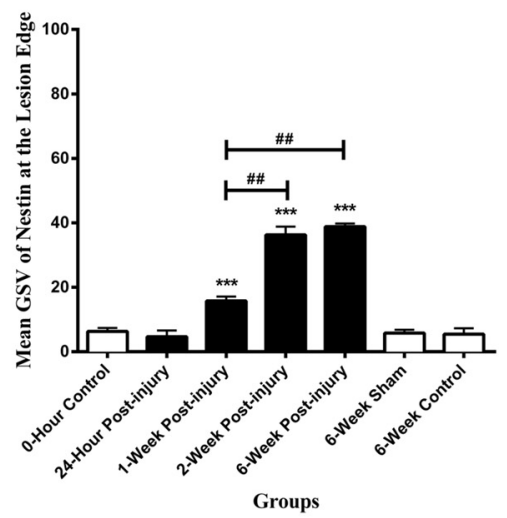

C

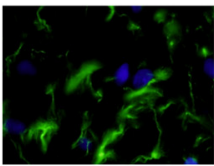

24-Hour Post-injury

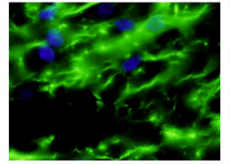

1-Week Post-injury

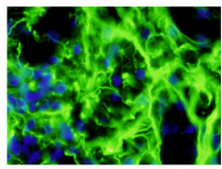

2-Week

Post-injury

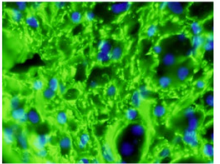

6-Week

Post-injury

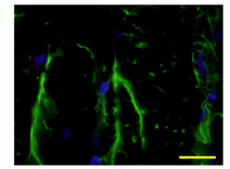

6-Week

Sham

D

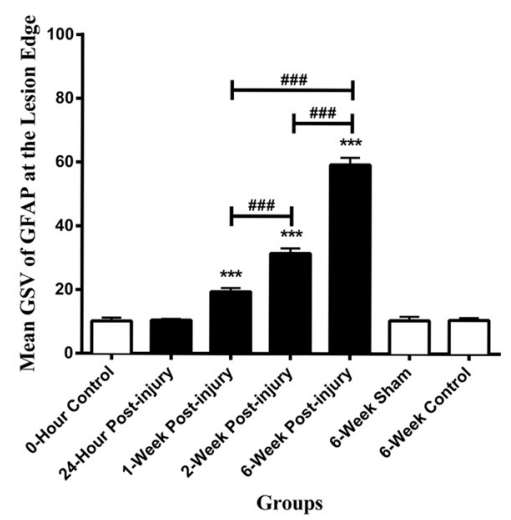

FIGURE 4 | Immunoreactivity of Nestin and GFAP at the lesion edge of rat spinal cord transverse sections. (A) Nestin positive cells were shown in red fluorescence, and the cell nuclei were counterstained in blue. Scale bar: $25 \mu \mathrm{m}$. (B) The Nestin positivity significantly elevated at 1-week post-injury and kept increasing to a maximum at 6 weeks post-injury. (C) GFAP positive cells were shown in green fluorescence, and the cell nuclei were counterstained in blue. Scale bar: $25 \mu \mathrm{m}$. (D) The GFAP positivity significantly elevated at 1-week post-injury and kept ascending to the maximum at 6 weeks post-injury. All data are presented as mean \pm SD. Statistically significant differences were shown compared to the 6 -week sham group $\left.{ }^{* * *} p<0.001\right)$. Statistically significant differences were revealed between groups $(\# \# p<0.01, \# \# p<0.001)$.

tended to be larger and had thicker processes than those seen in controls and in the surrounding tissue. It was difficult to identify individual cells due the overlap of cells and processes.

There were no significant differences in GFAP intensity in the rostral, epicenter and caudal sections so the average staining intensity for each spinal cord was used for all statistical analysis. The GFAP at the lesion edge was significantly increased from 1-week post-injury and continuously rose to the maximum at 6-week post-injury (Figure 4D). There were no significant differences in GFAP positivity between sham and control groups so the 6-week control group was used as a standard for subsequent analysis. 

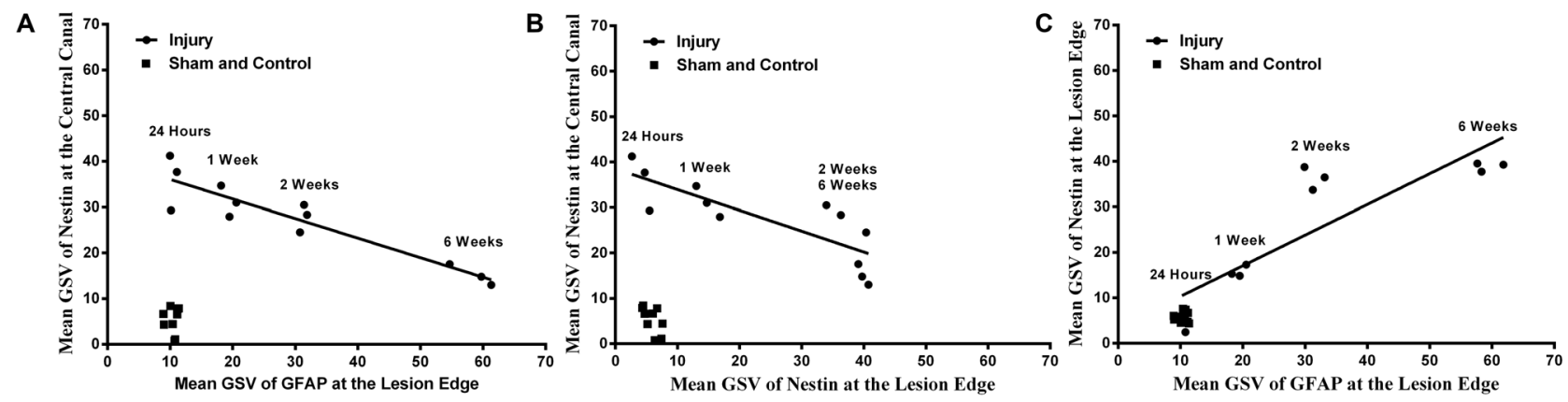

FIGURE 5 | Correlations between Nestin and GFAP immunoreactivity. Nestin immunoreactivity at the central canal was found to decline with (A) GFAP immunoreactivity at the lesion edge (Pearson's correlation, $r=-0.871, p<0.001$ ) and (B) Nestin immunoreactivity at the lesion edge (Pearson's correlation, $r=-0.786, p<0.001$ ) respectively. (C) Nestin immunoreactivity was proportionally increased at the lesion edge with the GFAP immunoreactivity at the lesion edge (Pearson's correlation, $r=0.900, p<0.001)$.

The GFAP immunoreactivity in 1-week, 2-week and 6week post-injury groups was significantly higher than that in 6-week control group respectively (Bonferroni post hoc comparisons, $p<0.001$ ). Moreover, the GFAP positivity in 6-week post-injury group was three-fold higher than that in 1-week injury group and two-fold larger than that in 2-week injury group (Bonferroni post hoc comparisons, $p<0.001)$.

\section{Nestin/GFAP Double Labeling}

The co-labeling of Nestin and GFAP was performed to determine whether the Nestin positive cells co-expressed GFAP (Figure 2). The ependymal cells of the central canal did not co-express Nestin and GFAP. However, in the injury groups there were many cells at the edge of the lesion were double labeled with GFAP and Nestin (Figure 2).

It was found that there was a strong negative correlation between Nestin immunoreactivity at the central canal and GFAP immunoreactivity at the lesion edge $(r=-0.926, p<0.001)$, as well as the Nestin immunoreactivity at the lesion edge $(r=-0.818, p<0.01$, Pearson's correlations; Figure 5). Furthermore, there was a directly proportional relationship found between Nestin and GFAP immunoreactivity at the lesion edge (Pearson's correlation, $r=0.867, p<0.001$ ).

\section{DISCUSSION}

The current study demonstrated the temporal response of endogenous NPCs following a mild SCI in rats. The contusion model described in this study led to the expected pattern of injury as reported by several groups (Basso et al., 1996; Mothe and Tator, 2005; Horky et al., 2006). At 24 h postinjury the tissue showed histological features consistent with an acute or primary SCI, for example, neutrophil infiltration, necrosis, hemeorrhage and axon swelling. By 6 weeks the tissue damage had developed into a cystic cavity (Li et al., 1999; Grossman et al., 2001; Westergren et al., 2001; Maier and Schwab, 2006).
The Nestin positive ependymal cells described in our study showed the well documented characteristics of NPCs reported by other groups (Frisén et al., 1995; Meletis et al., 2008; Cizkova et al., 2009; Barnabé-Heider et al., 2010). The up-regulated Nestin immunoreactivity in the ependymal layer of the central canal after injury has also been previously described (Yamamoto et al., 2001; Azari et al., 2005; Mothe and Tator, 2005; Cizkova et al., 2009). This population of cells at the central canal were Nestin positive and GFAP negative indicating NPCs rather than activated astrocytes (Lin et al., 1995; Johansson et al., 1999; Namiki and Tator, 1999). Although Nestin was not shown in control groups previously (Namiki and Tator, 1999; Mothe and Tator, 2005), we did see very occasional Nestin positive ependymal cells in the sham and control groups, suggesting that a limited number of quiescent endogenous NPCs do reside in the spinal cord. They probably function in maintaining homeostatic turnover of neural cells in normal adult spinal cords (Shibuya et al., 2002; Blasko et al., 2012). There were also a limited number of Nestin positive cells lining the blood vessels in the normal spinal tissue (Mokrý et al., 2004).

In our study Nestin staining was found to be highest in the ependymal cells lining the central canal at $24 \mathrm{~h}$ and then gradually declined over time. This is similar to the peak time reported previously by some authors (Namiki and Tator, 1999; Horky et al., 2006) but earlier than the peak at 3-7 days reported by others (Shibuya et al., 2002; Mothe and Tator, 2005; Cizkova et al., 2009; Hofstetter et al., 2005; Foret et al., 2010). It may be these authors were measuring specific populations of NPCs in different areas of the ependymal layer and subependymal regions. In our study, we were unable to label proliferating cells specifically with either Bromodeoxyuridine (BrdU) or Ki67 and therefore could not determine whether increases in Nestin staining was due to cell proliferation and migration or increased Nestin expression within individual cells. We did not observe large numbers of Nestin positive cells in the paranchyma adjacent to the central canal at the early time points, nor were there any differences in total ependymal 
cell numbers between the controls and SCI cords at any time, suggesting an increase in Nestin expression rather than an increase in cell number, or large numbers of migrating cells. Other authors are confident that the peak Nestin expression in ependymal cells in fact represents localized proliferation followed by migration from the central canal into the sub-ependymal area. This migration has been shown by Mothe and Tator (2005) who intraventricularly inject $1,1^{\prime}$-dioctadecyl6,6'-di(4-sulfophenyl)-3,3,3',3'-tetramethylindocarbocyanine to label ependymal cells for monitoring their migration along with the immunohistochemistry staining of BrdU and Ki67 to detect their proliferation. This was also supported by Barnabé-Heider et al. (2010) who used BrdU to label the proliferating cells in transgenic mice for tracking the ependymal cells, and Horky et al. (2006) who utilized retroviral vectors to trace the migration of endogenous NPCs in conjunction with $\mathrm{BrdU}$ for examining their proliferation status. In the studies that showed cell proliferation (Yamamoto et al., 2001; Mothe and Tator, 2005; Cizkova et al., 2009) it can be seen that there were relatively few BrdU positive nuclei in ependymal cells that are actually also Nestin positive indicating that while cell proliferation occurs there is also a marked up-regulation of Nestin protein in the non-proliferating ependymal cells post-injury, which is similar to what we found.

Astrogliosis was indicated by the increase in intensity of GFAP staining and by hypertrophy of astrocyte processes at the lesion edge (Pekny et al., 2014). In the current study astrogliosis was shown to increase above baseline levels by 1 week postinjury and steadily increase for the 6-week post-injury period, as has been previously decsribed (Frisén et al., 1995; Horky et al., 2006). Recent studies have suggested that astrogliosis forms as early as 3-4 days post-injury (Tian et al., 2007; TysselingMattiace et al., 2008). However, we could not confirm this, due to lack of post-injury time points between 1 day and 1 week.

Recently, reactive astrocytes, an important element of astrogliosis, were recognized by double labeling of GFAP and Nestin, which demonstrated the capability of reactive astrocytes to actively proliferate (Shibuya et al., 2002; Kozlova, 2003; Faulkner et al., 2004; Barnabé-Heider et al., 2010; Hu et al., 2010). As all the Nestin positive cells at the lesion edge co-expressed GFAP, Nestin immunoreactivity was utilized to investigate the response of reactive astrocytes following SCI in our study. The reponse of reactive astrocytes was not detectable at $24 \mathrm{~h}$ post-SCI, as shown in the early studies (Frisén et al., 1995; Shibuya et al., 2002). The maxium response of reactive astrocytes at the lesion edge was seen at 2 and 6 weeks post-injury as supported by Frisén et al. (1995). However, Shibuya et al. (2002) observed the strongest response of reactive astrocytes at 1 week post-injury, as a result of the examination of reactive astrocytes across the whole spinal cord transverse sections. Although from the traditional perspective, the glial scar largely composed of astrocytes was detrimental for neuroregeneration by preventing remyelination and regrowth of axons, the reactive astrocytes in glial scar were found to be beneficial for neural repair (Faulkner et al.,
2004; Okada et al., 2006; Wang et al., 2011). As has been well documented, the reactive astrocytes in the glial scar lining the lesion edge separated the damaged tissue, resulting in the restriction of inflammation and oedema spreading to intact tissue (John et al., 2003; Faulkner et al., 2004; Okada et al., 2006). An earlier study also suggested that reactive astrocytes played a protective role by preventing the accumulation of extracellular glutamate, released from injured neurons, which could damage surrounding healthy neural cells (Bush et al., 1999). Conversely, Ikeda and Murase (2004) argued that the reactive astrocytes were adverse for neuroregeneration by generating and secreting nitric oxide, a neurotoxin that damaged local neural cells contributing to the secondary injury (Kozlova, 2003; Barnabé-Heider et al., 2010). The reactive astrocytes in astrogliosis were also reported to inhibit axonal regeneration and oligodendrocytes differentiation from precursor cells, leading to remyelination failure (Ikeda and Murase, 2004; Hu et al., 2010; Wang et al., 2011).

Several authors have suggested that NPCs at the central canal upregulate Nestin expression and migrate towards the lesion edge where they differentiate into activated astrocytes and contribute to the glial scar (Mothe and Tator, 2005; Horky et al., 2006; Ke et al., 2006). Furthermore, BarnabéHeider et al. (2010) analyzed the origin of newly formed astrocytes at 2 weeks and 4 months post-SCI via transgenic mice and concluded that new astrocytes in the injured area came from both endogenous NPCs and astrocyte self-renewal. Foret et al. (2010) observed the increased GFAP expression in endogenous NPCs in the sub-ependymal area of the central canal 1 week after SCI, suggesting that the endogenous NPCs derived from the central canal mainly differentiated into astrocytes. While we saw the correlations that both Nestin and GFAP were increased at the lesion edge over the same time period, and there were many co-labeled cells, we did not see any evidence in the current study to suggest a direct result of migration and differentiation of NPCs from the central canal.

The current study agrees with the majority of the literature that NPCs located at the central canal are upregulated very early after SCI, certainly within 1 week, and most likely within $24 \mathrm{~h}$, as shown by the Nestin immunoreactivity. The astrocyte response at the injury site, as indicated by increased GFAP and Nestin staining occurs from 1 week post-injury and continues for at least 6 weeks. Many studies concur that a reduction in astrogliosis with a simultaneous survival of neurons will be beneficial to spinal cord repair and recovery. The sequence of cellular responses and the time frame shown in this study indicate that if a reduction in astrogliosis is to be targeted as part of a treatment strategy there is a relatively short window of opportunity to attempt cellular manipulation. Whether NPCs migrate from the central canal and differentiate into astrocytes, or whether the astrocytic reaction is a localized response at the lesion site it remains true that there is only a short time frame in which to alter or reduce astrogliosis. The question of how this might be managed in the context of an acute SCI remains to be answered and there is still a need for a viable strategy to circumvent the 
inflammatory cascade that occurs during at the same time period.

\section{AUTHOR CONTRIBUTIONS}

YM participated in the animal surgeries, completed all of the immunohistology, imaging and analysis. He undertook most of the manuscript preparation. KM participated in the animal surgeries and preparation of tissue for histology and immunohistochemistry, and assisted in manuscript preparation. CAG undertook the animal surgeries and supervised all other

\section{REFERENCES}

Azari, M., Profyris, C., Zang, D., Petratos, S., and Cheema, S. (2005). Induction of endogenous neural precursors in mouse models of spinal cord injury and disease. Eur. J. Neurol. 12, 638-648. doi: 10.1111/j.1468-1331.2005. 01066.x

Barnabé-Heider, F., Göritz, C., Sabelström, H., Takebayashi, H., Pfrieger, F. W., Meletis, K., et al. (2010). Origin of new glial cells in intact and injured adult spinal cord. Cell Stem Cell 7, 470-482. doi: 10.1016/j.stem.2010. 07.014

Barreiro-Iglesias, A. (2010). Targeting ependymal stem cells in vivo as a noninvasive therapy for spinal cord injury. Dis. Model. Mech. 3, 667-668. doi: 10. 1242/dmm.006643

Basso, D. M., Beattie, M. S., and Bresnahan, J. C. (1996). Graded histological and locomotor outcomes after spinal cord contusion using the NYU weight-drop device versus transection. Exp. Neurol. 139, 244-256. doi: 10.1006/exnr.1996. 0098

Blasko, J., Martoncikova, M., Lievajova, K., Saganova, K., Korimova, A., and Racekova, E. (2012). Regional differences of proliferation activity in the spinal cord ependyma of adult rats. Open Life Sci. 7, 397-403. doi: 10.2478/s11535012-0027-2

Bush, T. G., Puvanachandra, N., Horner, C. H., Polito, A., Ostenfeld, T., Svendsen, C. N., et al. (1999). Leukocyte infiltration, neuronal degeneration, and neurite outgrowth after ablation of scar-forming, reactive astrocytes in adult transgenic mice. Neuron 23, 297-308. doi: 10.1016/s0896-6273(00) 80781-3

Cawsey, T., Duflou, J., Weikert, C. S., and Gorrie, C. A. (2015). Nestin positive ependymal cells are increased in the human spinal cord after traumatic CNS injury. J. Neurotrauma 32, 1393-1402. doi: 10.1089/neu.2014.3575

Cizkova, D., Nagyova, M., Slovinska, L., Novotna, I., Radonak, J., Cizek, M., et al. (2009). Response of ependymal progenitors to spinal cord injury or enhanced physical activity in adult rat. Cell. Mol. Neurobiol. 29, 999-1013. doi: 10. 1007/s10571-009-9387-1

DeVivo, M. (2007). Sir Ludwig Guttmann Lecture: trends in spinal cord injury rehabilitation outcomes from model systems in the United States: 1973-2006. Spinal Cord 45, 713-721. doi: 10.1038/sj.sc.3102026

Dromard, C., Guillon, H., Rigau, V., Ripoll, C., Sabourin, J. C., Perrin, F. E., et al. (2008). Adult human spinal cord harbors neural precursor cells that generate neurons and glial cells in vitro. J. Neurosci. Res. 86, 1916-1926. doi: 10.1002/jnr. 21646

Faulkner, J. R., Herrmann, J. E., Woo, M. J., Tansey, K. E., Doan, N. B., and Sofroniew, M. V. (2004). Reactive astrocytes protect tissue and preserve function after spinal cord injury. J. Neurosci. 24, 2143-2155. doi: 10. 1523/jneurosci.3547-03.2004

Faulkner, S. D., Ruff, C. A., and Fehlings, M. G. (2013). The potential for stem cells in cerebral palsy-piecing together the puzzle. Semin. Pediatr. Neurol. 20, 146-153. doi: 10.1016/j.spen.2013.06.002

Foret, A., Quertainmont, R., Botman, O., Bouhy, D., Amabili, P., Brook, G., et al. (2010). Stem cells in the adult rat spinal cord: plasticity after injury and treadmill training exercise. J. Neurochem. 112, 762-772. doi: 10.1111/j.14714159.2009.06500.x aspects of the study including tissue analysis and manuscript preparation.

\section{ACKNOWLEDGMENTS}

We would like to acknowledge the staff at the Ernst Facility, University of Technology Sydney for taking care of all the experimental animals. We also thank the Microbial Imaging Facility, University of Technology Sydney for the fluorescent microscope and associated softwares. This work was supported by University of Technology Sydney Early Career Research Grant to $\mathrm{CAG}$.

Frisén, J., Johansson, C. B., Török, C., Risling, M., and Lendahl, U. (1995). Rapid, widespread, and longlasting induction of nestin contributes to the generation of glial scar tissue after CNS injury. J. Cell Biol. 131, 453-464. doi: 10.1083/jcb. 131.2.453

Grossman, S., Rosenberg, L., and Wrathall, J. (2001). Temporal-spatial pattern of acute neuronal and glial loss after spinal cord contusion. Exp. Neurol. 168, 273-282. doi: 10.1006/exnr.2001.7628

Guo, Y., Liu, S., Zhang, X., Wang, L., Zhang, X., Hao, A., et al. (2014). Sox11 promotes endogenous neurogenesis and locomotor recovery in mice spinal cord injury. Biochem. Biophys. Res. Commun. 446, 830-835. doi: 10.1016/j.bbrc. 2014.02.103

Hamilton, L. K., Truong, M. K., Bednarczyk, M. R., Aumont, A., and Fernandes, K. J. (2009). Cellular organization of the central canal ependymal zone, a niche of latent neural stem cells in the adult mammalian spinal cord. Neuroscience 164, 1044-1056. doi: 10.1016/j.neuroscience.2009. 09.006

Hofstetter, C. P., Holmström, N. A., Lilja, J. A., Schweinhardt, P., Hao, J., Spenger, C., et al. (2005). Allodynia limits the usefulness of intraspinal neural stem cell grafts; directed differentiation improves outcome. Nat. Neurosci. 8, 346-353. doi: 10.1038/nn1405

Horky, L. L., Galimi, F., Gage, F. H., and Horner, P. J. (2006). Fate of endogenous stem/progenitor cells following spinal cord injury. J. Comp. Neurol. 498, 525-538. doi: 10.1002/cne.21065

Hu, R., Zhou, J., Luo, C., Lin, J., Wang, X., Li, X., et al. (2010). Glial scar and neuroregeneration: histological, functional and magnetic resonance imaging analysis in chronic spinal cord injury: laboratory investigation. J. Neurosurg. Spine 13, 169-180. doi: 10.3171/2010.3.SPINE09190

Ikeda, H., and Murase, K. (2004). Glial nitric oxide-mediated long-term presynaptic facilitation revealed by optical imaging in rat spinal dorsal horn. J. Neurosci. 24, 9888-9896. doi: 10.1523/JNEUROSCI.260804.2004

Johansson, C. B., Momma, S., Clarke, D. L., Risling, M., Lendahl, U., and Frisén, J. (1999). Identification of a neural stem cell in the adult mammalian central nervous system. Cell 96, 25-34. doi: 10.1016/s0092-8674(00) 80956-3

John, G. R., Lee, S. C., and Brosnan, C. F. (2003). Cytokines: powerful regulators of glial cell activation. Neuroscientist 9, 10-22. doi: 10.1177/10738584022 39587

Ke, Y., Chi, L., Xu, R., Luo, C., Gozal, D., and Liu, R. (2006). Early response of endogenous adult neural progenitor cells to acute spinal cord injury in mice. Stem Cells 24, 1011-1019. doi: 10.1634/stemcells.2005-0249

Kozlova, E. N. (2003). Differentiation and migration of astrocytes in the spinal cord following dorsal root injury in the adult rat. Eur. J. Neurosci. 17, 782-790. doi: 10.1046/j.1460-9568.2003.02518.x

Li, G. L., Farooque, M., Holtz, A., and Olsson, Y. (1999). Apoptosis of oligodendrocytes occurs for long distances away from the primary injury after compression trauma to rat spinal cord. Acta Neuropathol. 98, 473-480. doi: 10. 1007/s004010051112

Lin, R., Matesic, D., Marvin, M., Mckay, R., and Brüstle, O. (1995). Re-expression of the intermediate filament nestin in reactive astrocytes. Neurobiol. Dis. 2, 79-85. doi: 10.1006/nbdi.1995.0008 
Maier, I. C., and Schwab, M. E. (2006). Sprouting, regeneration and circuit formation in the injured spinal cord: factors and activity. Philos. Trans. R. Soc. Lond. B Biol. Sci. 361, 1611-1634. doi: 10.1098/rstb.2006.1890

Meletis, K., Barnabé-Heider, F., Carlén, M., Evergren, E., Tomilin, N., Shupliakov, O., et al. (2008). Spinal cord injury reveals multilineage differentiation of ependymal cells. PLoS Biol. 6:e182. doi: 10.1371/journal.pbio. 0060182

Mokrý, J., Cizková, D., Filip, S., Ehrmann, J., Österreicher, J., Kolár, Z., et al. (2004). Nestin expression by newly formed human blood vessels. Stem Cells Dev. 13, 658-664. doi: 10.1089/scd.2004.13.658

Mothe, A., and Tator, C. (2005). Proliferation, migration and differentiation of endogenous ependymal region stem/progenitor cells following minimal spinal cord injury in the adult rat. Neuroscience 131, 177-187. doi: 10.1016/j. neuoscience.2004.10.011

Mothe, A. J., Zahir, T., Santaguida, C., Cook, D., and Tator, C. H. (2011). Neural stem/progenitor cells from the adult human spinal cord are multipotent and self-renewing and differentiate after transplantation. PLoS One 6:e27079. doi: 10.1371/journal.pone.0027079

Namiki, J., and Tator, C. H. (1999). Cell proliferation and nestin expression in the ependyma of the adult rat spinal cord after injury. J. Neuropathol. Exp. Neurol. 58, 489-498. doi: 10.1097/00005072-199905000-00008

Norenberg, M. D., Smith, J., and Marcillo, A. (2004). The pathology of human spinal cord injury: defining the problems. J. Neurotrauma 21, 429-440. doi: 10. 1089/089771504323004575

Okada, S., Nakamura, M., Katoh, H., Miyao, T., Shimazaki, T., Ishii, K., et al. (2006). Conditional ablation of Stat 3 or Socs 3 discloses a dual role for reactive astrocytes after spinal cord injury. Nat. Med. 12, 829-834. doi: 10.1038/nprot. 2006.155

Pekny, M., Wilhelmsson, U., and Pekna, M. (2014). The dual role of astrocyte activation and reactive gliosis. Neurosci. Lett. 565, 30-38. doi: 10.1016/j.neulet. 2013.12.071

Sakakibara, A., Aoki, E., Hashizume, Y., Mori, N., and Nakayama, A. (2007). Distribution of nestin and other stem cell'related molecules in developing and diseased human spinal cord. Pathol. Int. 57, 358-368. doi: 10.1111/j.1440-1827. 2007.02108.x

Shibuya, S., Miyamoto, O., Auer, R., Itano, T., Mori, S., and Norimatsu, H. (2002). Embryonic intermediate filament, nestin, expression following traumatic spinal cord injury in adult rats. Neuroscience 114, 905-916. doi: 10.1016/s03064522(02)00323-8

Thuret, S., Moon, L. D., and Gage, F. H. (2006). Therapeutic interventions after spinal cord injury. Nat. Rev. Neurosci. 7, 628-643. doi: 10.1038/nrn1955
Tian, D.-S., Dong, Q., Pan, D.-J., He, Y., Yu, Z.-Y., Xie, M.-J., et al. (2007). Attenuation of astrogliosis by suppressing of microglial proliferation with the cell cycle inhibitor olomoucine in rat spinal cord injury model. Brain Res. 1154, 206-214. doi: 10.1016/j.brainres.2007.04.005

Tysseling-Mattiace, V. M., Sahni, V., Niece, K. L., Birch, D., Czeisler, C., Fehlings, M. G., et al. (2008). Self-assembling nanofibers inhibit glial scar formation and promote axon elongation after spinal cord injury. J. Neurosci. 28, 3814-3823. doi: 10.1523/JNEUROSCI.0143-08.2008

Tzeng, S.-F. (2002). Neural progenitors isolated from newborn rat spinal cords differentiate into neurons and astroglia. J. Biomed. Sci. 9, 10-16. doi: 10. $1159 / 000048194$

Vessal, M., Aycock, A., Garton, M. T., Ciferri, M., and Darian-Smith, C. (2007). Adult neurogenesis in primate and rodent spinal cord: comparing a cervical dorsal rhizotomy with a dorsal column transection. Eur. J. Neurosci. 26, 2777-2794. doi: 10.1111/j.1460-9568.2007.05871.x

Wang, Y., Cheng, X., He, Q., Zheng, Y., Kim, D. H., Whittemore, S. R., et al. (2011). Astrocytes from the contused spinal cord inhibit oligodendrocyte differentiation of adult oligodendrocyte precursor cells by increasing the expression of bone morphogenetic proteins. J. Neurosci. 31, 6053-6058. doi: 10 1523/JNEUROSCI.5524-09.2011

Welkowitz, J., Cohen, B. H., and Lea, R. B. (2012). Introductory Statistics for the Behavioral Science. New York: John Wiley \& Sons.

Westergren, H., Farooque, M., Olsson, Y., and Holtz, A. (2001). Spinal cord blood flow changes following systemic hypothermia and spinal cord compression injury: an experimental study in the rat using Laser-Doppler flowmetry. Spinal Cord 39, 74-84. doi: 10.1038/sj.sc.3101127

Yamamoto, S.-I., Yamamoto, N., Kitamura, T., Nakamura, K., and Nakafuku, M. (2001). Proliferation of parenchymal neural progenitors in response to injury in the adult rat spinal cord. Exp. Neurol. 172, 115-127. doi: 10.1006/exnr.2001. 7798

Conflict of Interest Statement: The authors declare that the research was conducted in the absence of any commercial or financial relationships that could be construed as a potential conflict of interest.

Copyright (c) 2016 Mao, Mathews and Gorrie. This is an open-access article distributed under the terms of the Creative Commons Attribution License (CC BY). The use, distribution and reproduction in other forums is permitted, provided the original author(s) or licensor are credited and that the original publication in this journal is cited, in accordance with accepted academic practice. No use, distribution or reproduction is permitted which does not comply with these terms. 